\title{
Video mosaic watermarking using plasma key
}

\author{
Nidaa Flaih Hassan, Akbas Ezaldeen Ali, Teaba Wala Aldeen, Ayad Al-Adhami \\ Department of Computer Science, University of Technology, Iraq
}

\begin{tabular}{l} 
Article Info \\
\hline Article history: \\
Received Jun 10, 2020 \\
Revised Mar 16, 2021 \\
Accepted Apr 27, 2021 \\
\hline Keywords: \\
Attacks \\
Digital watermarking \\
Mosaic watermarking \\
Plasma effect \\
Video watermarking
\end{tabular}

Corresponding Author:

Ayad Aladhami

Department of Computer Science

University of Technology, Baghdad, Iraq

Email: 10010@uotechnology.edu.iq

\begin{abstract}
Video watermarking is one of the most widespread techniques amongst the many watermarking techniques presently are used; this is because the extreme existences of copyright abuse and misappropriation occur for video content. In this paper, a new watermarking algorithm is proposed to embed logo in digital video for copyright protection. To make the watermarks more robust to attack, host frame and host embedding indices must be changeable. A new algorithm is proposed to determined host frames by plasma function, Host location indices in frames are also determined by another plasma function. Logo is divided using the mosaic principle, the size of mosaic blocks is determined initially according to the degree of protection, whenever the size of mosaic blocks is small, it leads to safe embedding, and vice versa. Digital watermarks are embedded easily without any degradation for video quality, In the other side, the watermarked is retrieved by applying the reverse of proposed embedding algorithm and extracted watermark is still recognizable. The experimental results confirm that watermark is robust against three types of attacks which are addition of gaussian noise, JPEG compression, and rotation process.
\end{abstract}

This is an open access article under the CC BY-SA license.

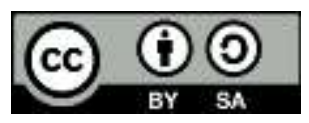

\section{INTRODUCTION}

The representation of electronic and the transformation of digital multimedia info (text, image, audio and video) causes an increase in theft, and the possibility of misuse of such info, in addition of increase of problems related with imposing copyrights on multimedia info [1]. Using internet and transmitting media over the network results in easy copying and distributing multimedia data over it. Digital watermarking is an alternate solution to keep the copyright of multimedia data [2].

Digital watermarking field was born in the same interval of the growth of world wide web. Digital watermarking is increasingly applied to hide information in digital multimedia; thus, the watermarking technology is applied against intellectual property human rights breach [3]. The digital watermarking is used to embed information in digital signal host with unnoticeable changes in signal. Digital watermarking has impending for covert transport network, finger-printing, signal- tagging, and control of media copyright [4].

Through the Internet, people can copy, process, and transmit videos of interest at will, which brings great convenience to people [5]. The speedy growth of multimedia requests, with distribution of large amount of video data via the internet, video protection becomes essential task. This can be done using video watermarking technology [6], thus in the last era, watermarking has become extensive in defensive the copyrights and authentication [2].

Video watermarking technology is a group of very significant technologies, its significance lies on effectiveness in copyright of digital video authentication and protection in the realization of the network 
environment. Facing the communication networks popularity of e-commerce, copyright protection of digital video, authentication difficulties and safe broadcast of digital info is the greatest vital difficult which is essential to be resolved. Technology of digital video watermarking is an operative and most capable technology to solve these difficulties [7].

Watermarking can be exposed to several kinds of attacks; hence there are some concerns, such as imperceptibility, capability, robustness to attacks and safety of the watermark that the watermarking requirements can be evaluated through the suggestion of a watermarking process [8]. For advanced flexibility to any alterations digital video file format, the built in information have to be equally dispersed through stream of video to decrease destructive chance for many number of bits of watermark all at once. Besides, it is essential to employment such methods for creating digital watermark that permit returning it by use of partial data [9].

The rest of this paper contains: Section 2 presents some prevailing video watermarking. Section 3 introduces digital video watermark. Effect of plasma is illustrated in Section 4. In Section 5 the proposed algorithm of watermarking is specified first, with embedding and extraction module description. Results and discussion are presented in Section 6. Section 7 presents the conclusion.

\section{RELATED WORKS}

Several different video watermarking techniques have been proposed, each technique focusing on a specific component, in the following section a brief description of watermarked techniques which are published recently:

X. Junxiaoet et al. [7] presented an algorithm for digital video watermarking using H.264, the proposed algorithm used the precise features of the compression standard H.264. Embedded watermark is randomly localized into the original video to be more robust, the video compressed bitstreams file is decoded before embedding watermarking to result nearer entropy coding level and this made less computation to be suitable for real time applications. Besides, DCT transform is used for watermark embedding to reduced degradation in the compressed video bit stream. The watermark can be extracted without the presence of the original video sequence, thus watermarked is extracted blindly by using selected altered coefficient at the decoder.

In [10] A. Koz et al. proposed a watermark approach for free viewing video. Watermark is embedded into each frame of many views by abusing properties of spatial masking for human visual system, and the location and rotation of the virtual camera is assumed to be known, the proposed approach, the watermark is extracted from an arbitrarily produced virtual image successfully. Also the approach is extended to be adaptive with the state of position and rotation of unknown simulated camera, by analyzing the configuration of watermark transformations due to rendering operations of image, and based on results of analysis, estimation of camera position and homograph are suggested for the simulated camera. The simulation results promise a new direction for watermarking research.

O. Faragallah [11] using two influential mathematical transformations, singular value decomposition (SVD) and discrete wavelet transform (DWT) domain. In this proposal frames of transformed video by using two resolution levels of DWT. High and middle frequency bands (HH, LH and HL) were transformed by SVD transformed and the watermark was hidden in each band in diagonal matrix. normalized correlation (NC) coefficient is used to estimate the similarities between the watermark cover and the embedded watermark from watermarked frames of digital video. This method illustrates that a cascade of DWT and SVD methods made watermark is robust against rotation, scaling, cropping and compression attacks than the conventional DWT methods.

M. Asikuzzaman et al. [12], proposed blind video watermarking algorithm. To provide high quality watermarked video, the watermark is hidden in chrominance channel base on one level of the dual tree complex wavelet transform, which is the same key that was used for the embedding in extraction phase. The proposed algorithm is strong to rotation, up scaling, compression, and cropping. The watermark is extracted from the dual-tree complex wavelet transform in any levels, and this depends on the down-scaled version resolution of the watermarked frame, also its provided robustness against temporal synchronization attacks. Such as conversion in frame rate, the watermark extracted from frame information without needing key that used through watermark embedding.

D. Kaur and S. Jindal in [13] proposed a new semi blind for watermark in video by combing discrete wavelet transform (DWT) and singular value decomposition (SVD). Each frame of video is a host for embedded object of watermark, embedding in each frame offered strength against attacks such as dropping of frame, averaging of frame and lossy compression. The proposal evaluated by using several videos samples subjected to some attacks for strength and imperceptible. Observation of the suggested scheme yields resulted good robustness against many attacks and good imperceptibility. 
R. Ahuja and S. Bedi, in [14] presented watermark embedded into particular discrete cosine transform blocks, in high frequency coefficients through intra coding procedure. The choice is important since errors in selected blocks are less sensitive to human eyes. Thus, the perceptibility of video watermark did not degrade. The quality of vision is also preserved since the compensated motions are untouched through the whole watermarking procedure. Results of experimental shown that the procedure is not only strong to following attacks: re-compression, spatial synchronization (rotation and cropping), but its robust against temporal synchronization attacks such as: delete frame, insert frame, insert frame, swap between frames and average of frames.

In [15] R. Ahuja and S. Bedi adapted a technique which apply on moving picture expert groups standard (MPEG-2), DCT coefficients are choose rapid decoder frames for watermarking purpose. Candidate I-frames are selected to accomplish good perceptibility and strength. Candidate frame is determined by secret key which constructed by cryptographic procedure. Extracted watermark is done by using three keys, the first key is used to halt extraction procedure, and the two other two keys are used to demonstrate the scrambled watermark. The robustness is measured by estimate the spatial and temporal synchronization attacks. High robustness is done against video specific often occurs attacks, high watermark capacity obtained by hosted thousands of bits in one frame.

C. Venkata Narasimhulu [16] proposed an additive and non-blind video watermark; this proposal is needed in extraction and detection process of the existence of each frame of video cover. The video frame is extracted with watermark decomposition at the same time by using contourlet transformation. Singular values for frames are changed with singular values of appropriate scaling factor watermark. Dissimilar watermarks are combined in diverse scenes of video cover to improve the strength of attacks. Experimental results demonstrated that the combination of nonsubsampled contourlet transform (NSCT) and singular value decomposition (SVD) algorithms achieve good imperceptibility and robustness to many attacks such as rotation, salt and pepper noise and frame dropping.

El-Shafai et al. [17] presented two hybrid watermarking methods for three dimensional high efficiency video coding (3D-HEVC). Homomorphic transform based singular value decomposition (SVD) in discrete wavelet transform (DWT) domain is the first watermarking method, while the second watermarking method used three levels discrete stationary wavelet transform (DSWT) in discrete cosine transform (DCT) domain. These two adapted hybrid watermarking methods are accomplished high capacity, security, and strength without influence on perceptual quality of 3DV. Watermark frames are extracted with good quality.

H. Mareen, et al. [18] presented watermark in video in which explicitly changed a single encoder decision. Formerly, the explicit change automatically broadcasts into a great group of implied distortions that signifies the Watermark. The implied distortions ordinary formed artifacts in compression and imperceptibility. This paper results watermark forceful against video influences, besides, the proposed scheme has required no alteration of electronic devices consumer. Thus, the proposed watermarking could be used to help protect piracy without concerning innocent users with abnormal distortions.

In [19] S. Almuzairai and N. Innab proposed video watermarking system that embedded watermark in audio streams and the visual stream to guarantee the watermarked video integrity. The watermark is embedded in moving portions of the visual stream of the video using moving block detection (MBD) algorithm, MBD usages entropy to detect host moving portions visual stream. To ensure transparency and robustness against attacks, embedding process in visual stream is achieved by using DWT. Power factors of DWT is employed for controller transparency level. Also, silence deletion algorithm (SDA) is used embedding the watermark after eliminating the noise from original audio stream. To guarantee robustness against attacks DCT is used to embed the watermark. Using the geometric attacks, compression and the Gaussian noise non-geometric attacks, the experimental results proved that the proposed system outdid the following systems: I_frame, key_frame, LBS_based systems, and spread_spectrum.

Z. Cao and L. Wang [20] presented video watermarking algorithm used hyperchaotic lorentz system. Confidentiality improved by scramble watermark images using hyperchaotic lorentz system. Dhot detection is used to detect non motion frames of the video, and the host frames are selected by using chaotic arrangement. Wavelet transform is applied to selected frames to obtain proper sub bands for embedding watermark. The performance indicated high imperceptibility based on results obtained by applying peak signal-to-noise ratio, structural similarity index measure, and normalized correlation. Also robustness and imperceptibility are obtained under geometrical, image processing, and video attacks.

In [21] F. Layth Malallah et al. introduced watermarking in video based on arnold transform and integer wavelet transforms (IWT). IWT is used to de-compose the cover frames, and arnold transform is employed to scramble grey scale image watermark. Embedding made transmission more secure by disordering the information by scrambling the watermark before embedding. System performance is compared with some related video watermarking systems, in which the assessment procedures contain testing against some video processes and attacks. Accordingly, the system proved to be robust perfectly. 


\section{DIGITAL VIDEO WATERMARK}

With the hasty expansion of technologies of multimedia, and internet of things, people are giving more devotion to the safety of digital audio, images and videos [22]-[23]. Growing quantity of digital videos which are transferred shared and exchanged over the Internet causes an illegal copying and defective distribution of digital video content, and this considered serious problems [19].

Lately, in Internet multimedia applications video is considered as main part, such example of video applications: videophone, set-top box, video on demand and video conference, these applications are needed to be automatically secured to protect them from planned and unplanned attacks. As an effective secured component, video watermarking is considered as vital component which can be used to enhance privacy and security of these applications. Essentially, watermark in video is considered as enhancement type of watermark in image, watermark could be hided the video straight or in the transformed form of host video. Digital video is a sequence of successive still images identified as frames, but watermark in video identified problems of large capacity, additionally, video watermarking must robust to additional attacks counting: frame averaging, frame swapping, video coding and video compression. Another concerned occupied with watermark in video is afford the balance between motion, and motionless regions [24].

\section{EFFECT OF PLASMA}

The effect of plasma is used frequently in demos to produce a shaky visual animated effect. Many ways are used to create such effects as animated plasmas. Principle of effect of plasma actually simple, in the past, plasma effects were created with a recomputed color table. These days, the same effect can be produced in real-time with sinusoids (sum of sine in y-direction and sine in x-direction) to grant an illusion of liquid and organic movement. No limits exist for the generation of plasma function, as an example, define a plasma function can be defined as a combination of the many functions: F1, F2, and F3. Each one of these function can apply Sine to the distance between any two points or to the value of current time [25].

\section{THE PROPOSED ALGORITHM}

Design of digital video watermarking algorithms presents an issue when it's compared with designing image watermarking algorithms. Unlike image watermarking, video consider as challenge to the designer, thus to optimize video watermarking embedding technology, the following issues are considered in this proposal, and they are:

1) Watermarked logo is divided based on mosaic division. Sizes of division are depend on layer of protection.

2) Watermark embedding location within the cover video is determined by two keys, one of these keys is used to determine the frame number, and the second key is used to determine the embedding location in frame, second key value is correspond to upper left corner of logo block. These keys are computed by using plasma equations agreed upon by the transmitting and receiving parties.

In order to preserve video copyrights, watermarking algorithm is proposed which consists of twomodules: (1) Embedding module and (2) Extracting module.

Figure 1 shows the main block of the suggested watermark in digital video.

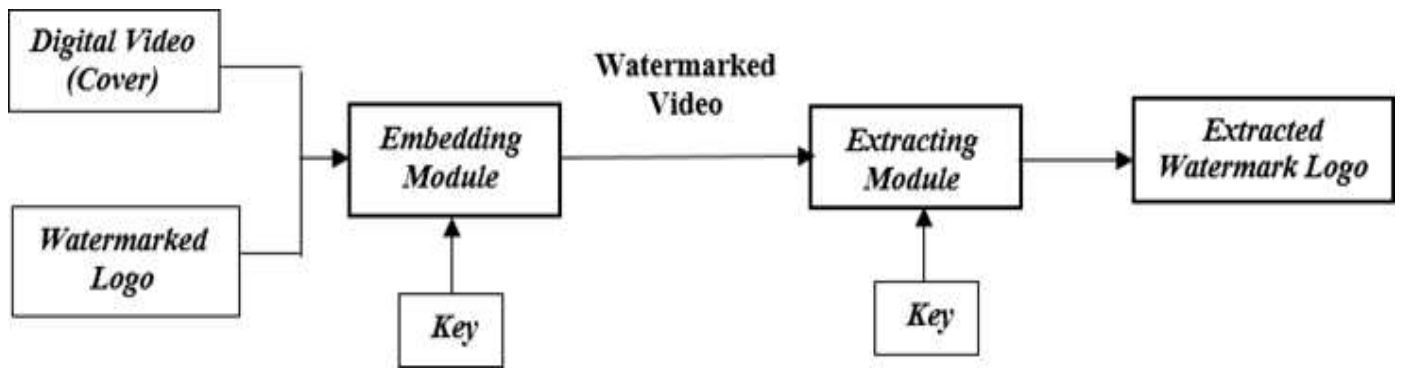

Figure 1. The proposed watermarking algorithm in digital video

A. Embedding module

Embedding module is at the sending end, it is achieved by extracted all frames from the hosted digital video sequence to be prepared for embedding logo block within video file. Before embedding, the 
logo image is divided into finite numbers of mosaic blocks. The number of blocks and their dimensions are determined according to the requirement of security degree. These blocks are inserted into the selected frames. The mosaic block dimensions can be in different size. It may be 1 pixel, $2 \times 2$ pixels, $3 \times 3$ pixels block, or any dimension can be determined by sender. Hosted frames are selected according to the output of plasma (1):

$$
\text { Current_Host_Frame }=2+\mathrm{w}+.5 * \operatorname{Sin}(\text { Time } / 5.0)
$$

Where $\mathrm{w}$ is a random numbers. The result from (1) must be an integer value that is equal or less than the number of frames in the hosted video. For this reason, the integer part of the resulted number will be taken only and neglects the fraction part, as an example; if the Host-frame equal to 2.7355, then the Current_ host_frame will be to (2). Hosted blocks locations in the video frames can be determined from the output of plasma (2):

$$
\text { Current_Host_Location }=127.5+(127.5 * \operatorname{Sin}((\mathrm{w} / 32)))+(127.5+(127.5 * \operatorname{Sin}(\mathrm{h} / 32)))
$$

Where $\mathrm{h}$ is a random numbers. The output from (2) will be divided into two parts: the integer part (left of floating-point) for $\mathrm{x}$-axis and the fraction part (right of the floating-point) for $\mathrm{y}$-axis of the coordinates. As an example if the hosted-location is equal to 262.967 then the new x will be equal to 262 , y is equal to 967 , and $(262,967)$ will be the coordinates of the location that the next block of the mosaic watermark must be located. Figure 2 shows the block diagram of embedding module, and Algorithm 1 describes the algorithm of embedding module.

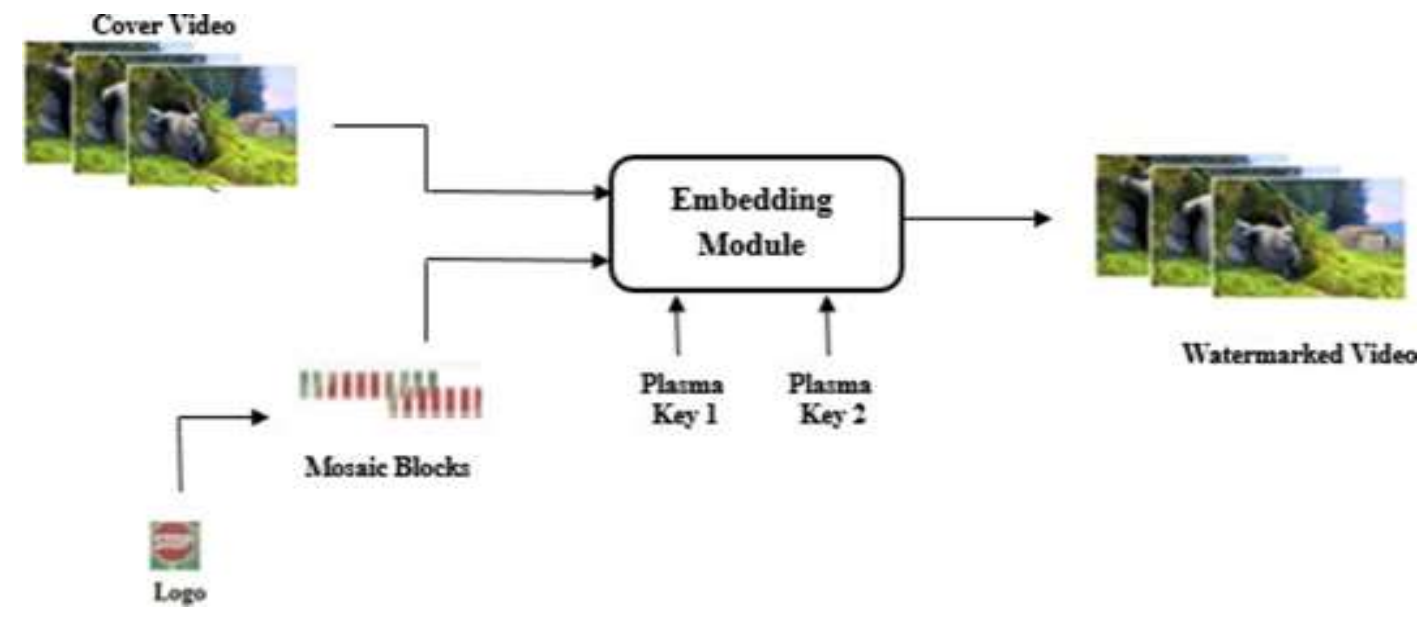

Figure 2. The proposed embedding module

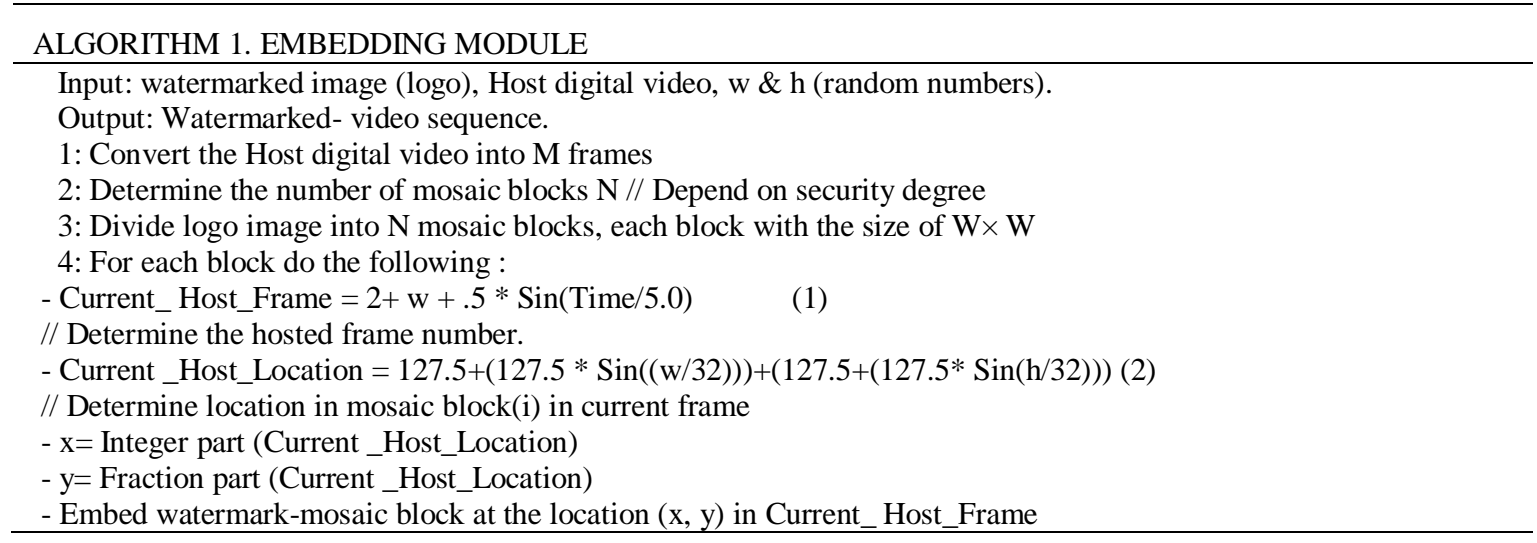




\section{B. Extraction Module}

Extracting is the reverse of the embedding process, and it's applied at the receiver side, where the logo image's mosaic blocks are extracted from the selected frames and collected to retrieve the original watermarked logo, Algorithm 2 illustrates extracting module.

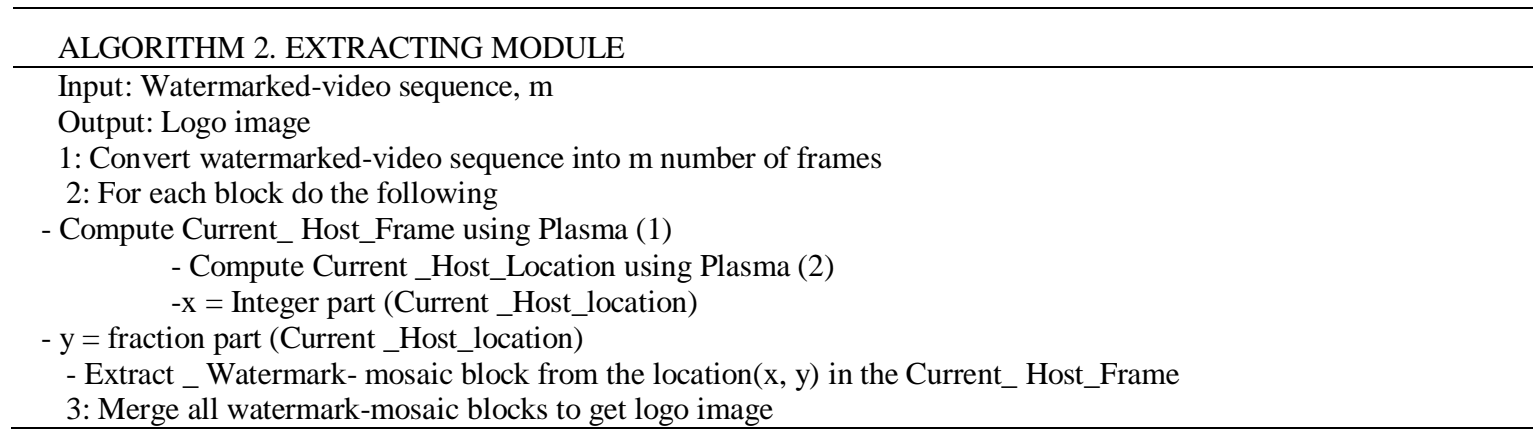

\section{RESULTS AND DISCUSSION}

Evaluation of performance is considered as an essential part in designing watermark algorithm, objective measures define the overall error between samples of input video files, and the corresponding samples of the watermarked video, thus they are used to evaluate the performance of the algorithm to state whether it's effective or not. Evaluation of this proposed algorithm is based on mean squared error (MSE), signal to noise ratio (SNR), peak signal to noise ratio (PSNR), and bit error ratio (BER) number and ratio [26]. These measures define the overall error between samples of input video files and the corresponding samples of the watermarked video. This kind of measure can be a good testing tool for quality. Two samples of digital video are used to declare evaluation results, the specification of these samples are declared in Table 1.

Table 2 list objective results (MSE, SNR, PSNR and BER) after embedding watermark logo in Video_1, first column declares size of logo block, last row of watermark block size represents whole watermark size embedded in one frame, Figure 3 shows an example of some test images from Video_1 before and after hiding a watermark. From the results listed in Table 1, it is noted that these metrics give good results for keeping the watermarked video transparent, where higher PSNR and lower MSE values imply closer resemblance between the watermarked and the original frame. Table 3 lists objective results (MSE, SNR, PSNR and BER) after embedding watermark logo in Video_2, Figure 4 shows an example of some test images from Video_2 before and after hiding a watermark.

Table 1. Specifications of video samples

\begin{tabular}{ccccc}
\hline Test Video Sample & Video File Type & Frame Dimensions $(\mathrm{W} \times \mathrm{H})$ & Frame Rate (frames/Second ) & No. of Frames \\
\hline Video_1 & $\mathrm{mp} 4$ & $320 \times 240$ & 30 & 20 \\
Video_2 & $\mathrm{mp} 4$ & $640 \times 368$ & 25 & 22 \\
\hline
\end{tabular}

Table 2. MSE, SNR, PSNR and BER after embedding watermark logo in Video_1

\begin{tabular}{ccccc}
\hline Watermark Block size & MSE & SNR & PSNR & BER Number BER Ratio \\
\hline $1 \times 1$ & 0.2210 & 52.7878 & 54.6877 & 231780.0175 \\
$3 \times 3$ & 0.5550 & 48.7883 & 50.6882 & 219240.0166 \\
$5 \times 5$ & 1.2734 & 45.1814 & 47.0813 & 224100.0169 \\
$8 \times 8$ & 4.5341 & 39.6660 & 41.5659 & 228560.0173 \\
$10 \times 10$ & 7.3844 & 37.5477 & 39.4476 & 222660.0168 \\
$30 \times 30$ & 57.6127 & 28.6257 & 30.5256 & 254150.0192 \\
\hline
\end{tabular}

Table 3. MSE, SNR, PSNR and BER after embedding watermark logo in Video_2

\begin{tabular}{ccccc}
\hline Watermark Block size & MSE & SNR & PSNR & BER Number BER Ratio \\
\hline $1 \times 1$ & 0.3386 & 50.0016 & 52.8344 & 858010.0285 \\
$3 \times 3$ & 0.6190 & 47.3808 & 50.2137 & 864370.0287 \\
$5 \times 5$ & 1.3342 & 44.0456 & 46.8785 & 874910.0290 \\
$8 \times 8$ & 2.8453 & 40.7567 & 43.5896 & 860470.0285 \\
$10 \times 10$ & 4.7778 & 38.5057 & 41.3385 & 872910.0290 \\
$30 \times 30$ & 46.5635 & 28.6175 & 31.4503 & 930870.0309 \\
\hline
\end{tabular}




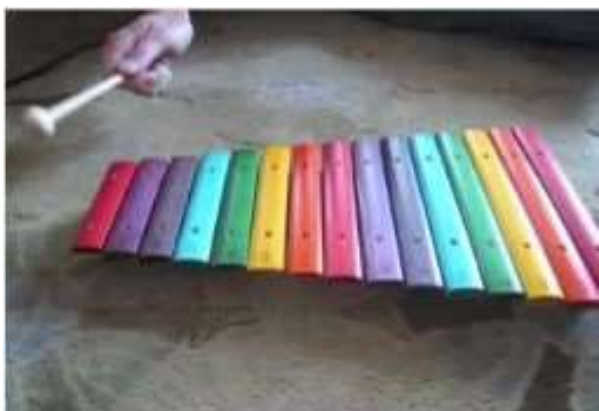

(a). Original frame

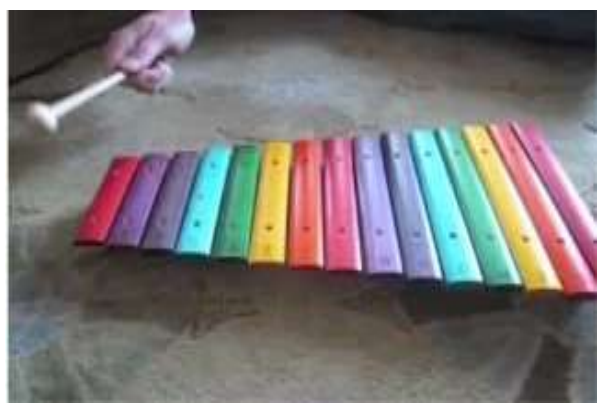

(c). Mosaic block is with $3 \times 3$ pixels

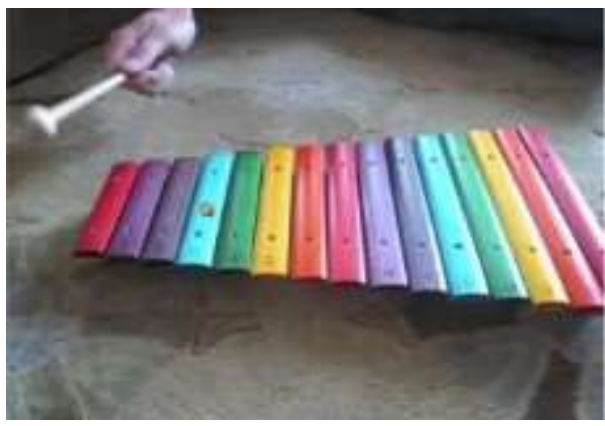

(e). Mosaic block is with $8 \times 8$ pixels

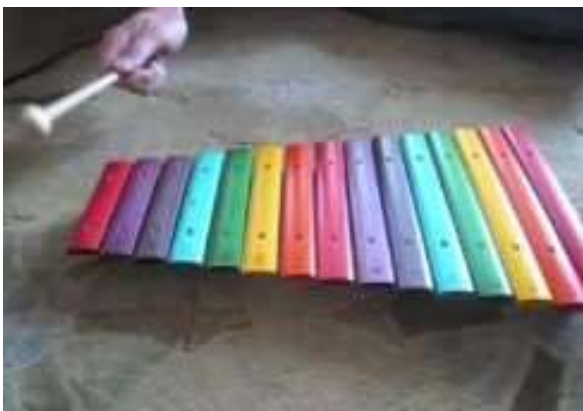

(b). Mosaic block is with 1- pixel

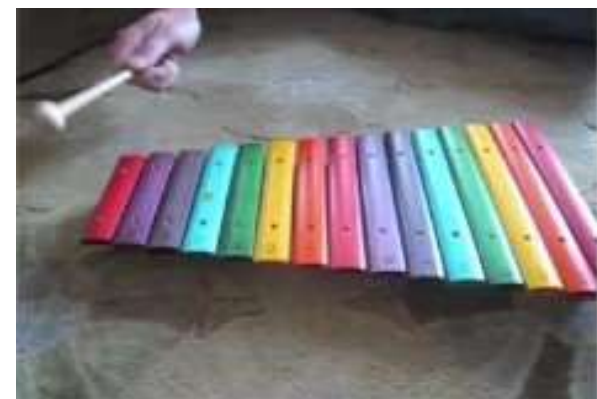

(d). Mosaic block is with $5 \times 5$ pixels

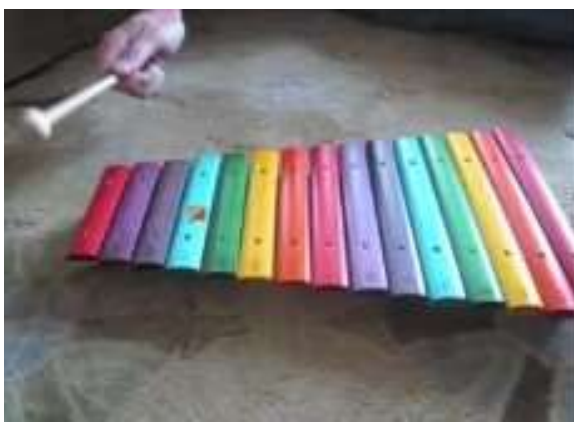

(f). Mosaic block is with $10 \times 10$ pixels

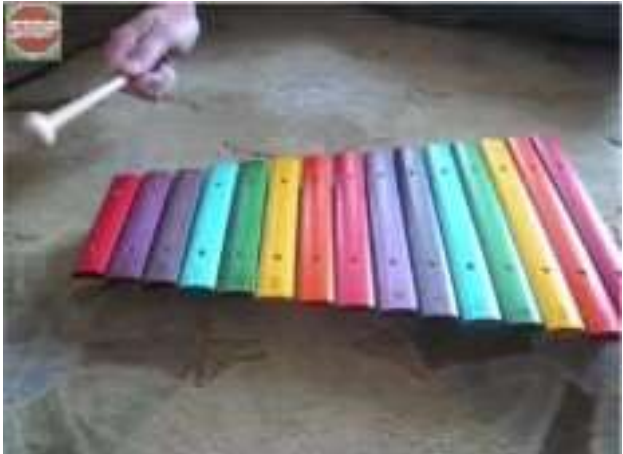

(g). All mosaic blocks are embedded in one frame at most top left corner

Figure 3. Test images from Video_1

Any watermarking scheme should have robustness against some attacks, watermark attack attempts to destruction the inserted watermark by alterations of the whole cover without any effort to detect and separate the watermark. In this proposal, fairly high robustness is achieved, by using three attacks, the addition of Gaussian noise, JPEG compression( lossy compression ) and rotation( geometric process), Table 4 shows Bit Per Pixel resulted from applied three mentioned attacks on two test video samples. Comparison with related work as shown in Table 5. 
There is proportional relationship between the security of the watermark and the time needs to implement its algorithm, whenever the numbers of the blocks are large, the block size will be small, and because they will be spread widely in the video, both of the watermark protection and the processing time of the algorithm will be increased. Then, the security against brute force attack will be more solid. Calculation of time complexity for the proposed algorithm of watermark depends on the total size of the watermark, the method of its division into blocks, and the plasma function complexity. In Big O Notation, its time complexity $=\mathrm{O}(\mathrm{N})$, where $\mathrm{N}=$ number of blocks. One of the good achievements of the proposed algorithm is that no possible for the naked eye to distinguish the difference between the original image and the one that is embedded with a watermark of block size of 1 pixel as illustrated in Figure 3(b) and Figure 4(b).

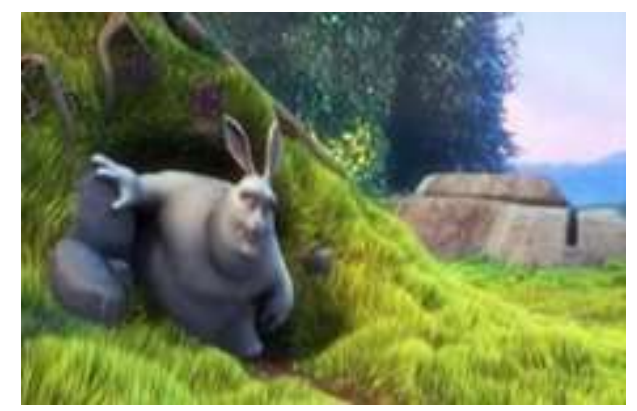

(a). Original frame

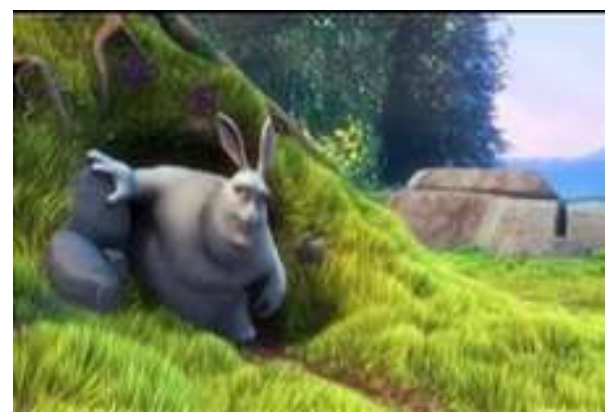

(c). Mosaic block is with $3 \times 3$ pixels

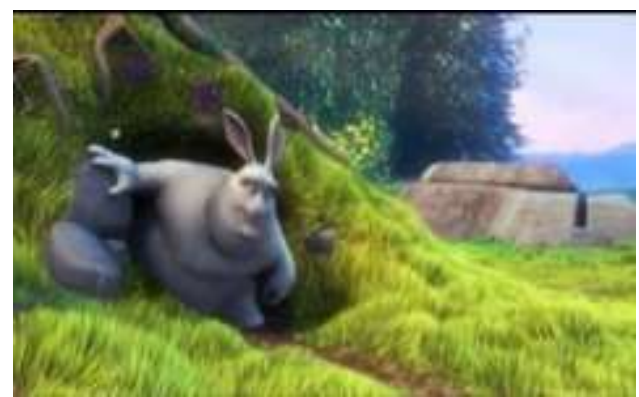

(e). Mosaic block is with $8 \times 8$ pixels

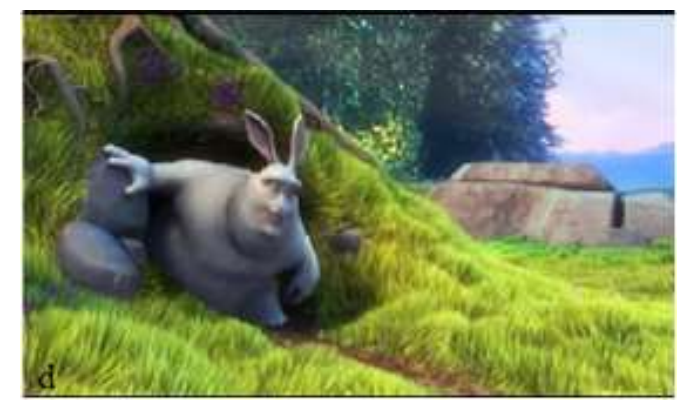

(b). Mosaic block is with 1- pixel

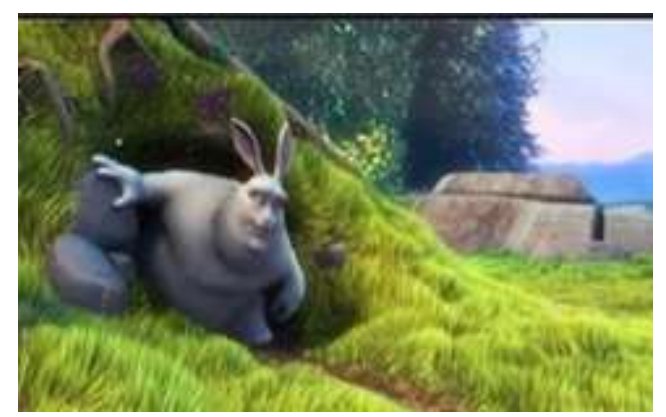

(d). Mosaic block is with $5 \times 5$ pixels

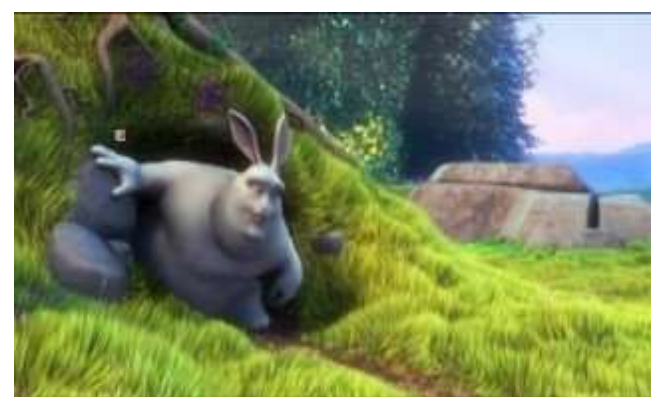

(f). Mosaic block is with $10 \times 10$ pixels

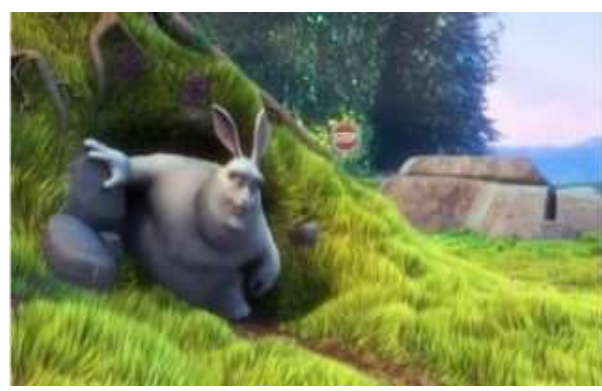

(g). All mosaic blocks are embedded in one frame at most top left corner

Figure 4. Test images from Video_2 
Table 4. Watermarked video frame performance parameter against three attacks

\begin{tabular}{ccc}
\hline Attack Types & BER Ratio Video_1 & BER Ratio Video_2 \\
\hline Gaussian noise $($ mean=0, variance =0.001) & 0.3254 & 0.2236 \\
JPEG Compression & 0.1126 & 0.1358 \\
Rotation & 0 & 0 \\
\hline
\end{tabular}

Table 5. Comparison with related work

\begin{tabular}{|c|c|c|c|}
\hline \multirow[t]{2}{*}{ Robustness against attack } & \multicolumn{2}{|c|}{ Existing Schemes } & \multirow[t]{2}{*}{ Proposed Schemes } \\
\hline & N. Alias \&F. Ernawan [2] & R. Ahuja and S. Bedi [15] & \\
\hline Gaussian noise & ****** & $* * * *$ & $* * * *$ \\
\hline JPEG Compression & $* * * * *$ & --- & $* * * * *$ \\
\hline Rotation & $* * *$ & $* * *$ & $* * * * *$ \\
\hline
\end{tabular}

\section{CONCLUSION}

In order to create watermarking robust against copyright infringement, this paper suggests a new color video watermarking technique. Logo is embedded in video based on two subjects : the first one; logo is divided using mosaic technique, whenever size of mosaic division is small, robustness of the watermarked is increased and made embedding process more safety. The second subject; two plasma functions are used to generate two random values periodically; these two values represent host frame and host location in frame. These two subjects made watermark in video robust against video manipulations. The experimental results show that the proposal watermark is robust against three addition of gaussian noise, JPEG compression and rotation process without any degradation to video cover and logo in extraction process. For future work, performance of the suggested algorithms can be improved by decreasing the execution time of video to be used for on-line video watermark.

\section{REFERENCES}

[1] E. Koch and J. Zhao, "Towards Robust and Hidden Image Copyright Labeling," Proc. of 1995 IEEE Workshop on Nonlinear Signal and Image Processing, Neos Marmaras, Greece, June 20-22, 1995.

[2] N. Alias and F. Ernawan, "Multiple watermarking technique using optimal threshold," Indonesian Journal of Electrical Engineering and Computer Science (IJEECS), vol. 18, no. 1, pp. 368-376, April 2020, doi: 10.11591/ijeecs.v18.i1.pp368-376.

[3] C. Busch et al., "Digital watermarking: From concepts to real-time video applications," IEEE Computer Graphics and Applications, Feb. 1999, doi: 10.1109/38.736466.

[4] P. Chan et al., "A Novel Scheme for Hybrid Digital Video Watermarking: Approach, Evaluation and Experimentation," IEEE Transactions on Circuits And Systems For Video Technology, vol. 15, no. 12, Dec. 2005, doi: 10.1109/TCSVT.2005.856932.

[5] X. Yu et al., "A Survey on Robust Video Watermarking Algorithms for Copyright Protection," Applied Sciences, Oct 2018, doi: 10.3390/app8101891.

[6] M. Koubaa et al., "Collusion-resistant video watermarking based on video mosaicing," Proceedings of the Eighth IEEE International Symposium on Multimedia (ISM'06), IEEE 2006, doi: 10.1109/ISM.2006.49.

[7] X. Junxiao et al., "A novel digital video watermarking algorithm," International Conference on Advances in Engineering, 2011, doi: 10.1016/j.proeng.2011.11.2607.

[8] M. Asikuzzaman and M. Pickering, "An Overview of Digital Video Watermarking," IEEE Transactions on Circuits and Systems for Video Technology, 2017, doi: 10.1109/TCSVT.2017.2712162.

[9] S. Belim and P. Cherepanov, "Digital video watermarking algorithm robust against video container format changes," IOP Conf. Series: Journal of Physics, 2019, doi: 10.1088/1742-6596/1260/2/022001.

[10] A. Koz et al.,"Watermarking of Free-view Video," IEEE Transactions on Image Processing, vol. 19, no. 7, Jul. 2010, doi.: 10.1109/TIP.2010.2045024.

[11] O. Faragallah, "Efficient video watermarking based on singular value decomposition in the discrete wavelet transform domain," AEU-International Journal of Electronics and Communications, vol. 67, no. 3, March 2013, pp. 189-196, doi: 10.1016/j.aeue.2012.07.010.

[12] M. Asikuzzaman, et al., "Imperceptible and Robust Blind Video Watermarking using Chrominance Embedding: A Set of Approaches in the DT CWT Domain," IEEE Transactions On Information Forensics And Security, August 2014. doi: 10.1016/j.aeue.2012.07.010.

[13] D. Thind and S. Jindal, "A Semi Blind DWT-SVD Video Watermarking," International Conference on Information and Communication Technologies (ICICT ), 2014, doi: 10.1016/j.procs.2015.02.104).

[14] R. Ahuja and S. Bedi, "Robust Video Watermarking Scheme Based on Intra-Coding Process in MPEG-2 Style," International Journal of Electrical and Computer Engineering (IJECE), vol. 7, no. 6, pp. 3332-3343, December 2017, doi: 10.11591/ijece.v7i6.pp3332-3343.

[15] R. Ahuja and S. Bedi, "Video Watermarking Scheme based on Candidates I-Frames for Copyright Protection," Indonesian Journal of Electrical Engineering and Computer Science (IJEECS), vol. 5, no. 2, pp. 391-400, Feb 2017, doi: 10.11591/ijeecs.v5.i2.pp391-400. 
[16] C. Venkata Narasimhulu, "A robust hybrid video watermarking algorithm using NSCT and SVD," IEEE International Conference on Power, Control, Signals and Instrumentation Engineering (ICPCSI), 2017, doi: 10.1109/ICPCSI.2017.8391961.

[17] W. El-Shafail et al., "Efficient multi-level security for robust 3D color-plus-depth HEVC," Springer Science Business Media, LLC, part of Springer Nature 2018. vol. 77, pp. 30911-30937, 2018. doi: 10.1007/s11042018-6036-z.

[18] H. Mareen et al., "A Novel Video Watermarking Approach Based on Implicit Distortions," Article in IEEE Transactions on Consumer Electronics, July 2018. doi: 10.1109/TCE.2018.2852258.

[19] S. Almuzairai and N. Innab, "Video Watermarking System for Copyright Protection based on Moving Parts and Silence Deletion," International Journal of Advanced Computer Science and Applications (IJACSA), vol. 10, no. 2, 2019, doi: 10.1109/ICPCSI.2017.8391961.

[20] Z. Cao and L. Wang, "A secure video watermarking technique based on hyperchaotic Lorentz system," Multimedia Tools and Applications, 78:26089-26109, 2019, doi: 10.1007/s11042-019-07809-5.

[21] F. Layth Malallah et al., "Reversible color video watermarking scheme based on hybrid of integer-to-integer wavelet transform and Arnold transform," International Journal of Electrical and Computer Engineering (IJECE), vol. 10, no. 4, pp. 3519-3527, August 2020, doi: 10.11591/ijece.v10i4.pp3519-3527.

[22] C. Wang et al., "Anti-HEVC Recompression Video Watermarking Algorithm Based on the All Phase Biorthogonal Transform and SVD," IETE Technical Review (Online) Journal, vol. 35, no. S1, pp. 42-58, doi: 10.1080/02564602.2018.1477631.

[23] C. Li et al., "A Semi-Fragile Video Watermarking Algorithm Based on H.264/AVC," Mobile Intelligence Assisted by Data Analytics and Cognitive Computing 2020, 2020, doi: 10.1155/2020/8848553.

[24] M. Nematollahi et al., "Digital Watermarking Techniques and Trends," Springer Topics in Signal Processing, 2017.

[25] A. Ali and N. Hassan, "Proposing a Scheme for Human Interactive Proof Test using Plasma Effect," Baghdad Science Journal, vol. 16, no. 2019.

[26] T. Dutta and H. Gupta, "A robust watermarking framework for high efficiency video coding (HEVC)encoded video with blind extraction process," Journal of Visual Communication and Image Representation, vol. 38, pp. 29-44, July 2016, doi: 10.1016/j.jvcir.2015.12.007.

\section{BIOGRAPHIES OF AUTHORS}

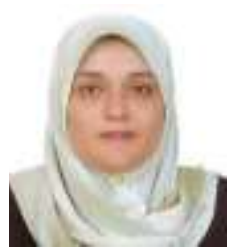

Nidaa Flaih Hassan received the MSc. and PhD. in Computer Science from the Computer Science Dept. University of Technology-Iraq. She earned MSc. In 1996 and PhD. In 2005. She has 25 years' experience in teaching field. Her areas of interest are data security, computer security, image processing, and network security.

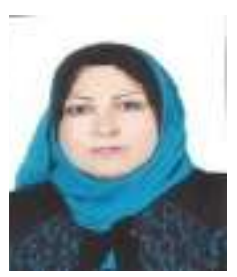

Akbas Ezaldeen received the MSc. and Ph.D. in computer science from the Department of Computer Science/University of Technology-Iraq in 1996 and 2016 respectively. I have 22 years of experience of teaching and my specialty is in the area of image and video processing.

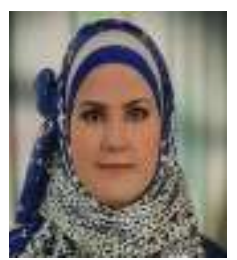

Teaba Wala Aldeen khairi received her B.Sc. and MSc. degrees in Software from Technology University, Baghdad, Iraq in 2005, 2015 respectively. Her research interests include Artificial Intelligence, Image Processing, and, Computer Vision.

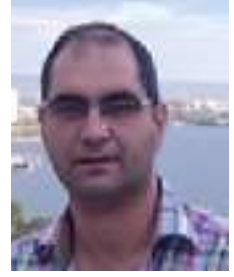

Dr. Ayad Al-Adhami received the MSc degree in 2008 and was specified in data security (Mathematics \& computer applications) From AlNahrain University, Iraq-Baghdad. The PhD degree earned in 2018 and specified in computing (Computer security) Plymouth University, United Kingdom. Areas of study are RFID security, Authentication \& privacy, Cryptography and Information security. 